\title{
Spatially-explicit estimation of Wright's neighborhood size in continuous populations
}

\author{
Andrew J. Shirk ${ }^{*}$ and Samuel A. Cushman ${ }^{2}$ \\ ${ }^{1}$ College of the Environment, Climate Impacts Group, University of Washington, Seattle, WA, USA \\ 2 USDA Forest Service, Rocky Mountain Research Station, Flagstaff, AZ, USA
}

\author{
Edited by: \\ Melanie April Murphy, University of \\ Wyoming, USA \\ Reviewed by: \\ Peter Poczai, University of Helsinki, \\ Finland \\ Margarida Matos, Faculdade de \\ Ciencias da Universidade de Lisboa, \\ Portugal \\ Juan Jose Robledo-Arnuncio \\ INIA-CIFOR, Spain \\ *Correspondence: \\ Andrew J. Shirk, College of the \\ Environment, Climate Impacts \\ Group, University of Washington, \\ Box 355674, Seattle, WA 98195, \\ USA \\ e-mail: ashirk@uw.edu
}

\begin{abstract}
Effective population size $\left(N_{e}\right)$ is an important parameter in conservation genetics because it quantifies a population's capacity to resist loss of genetic diversity due to inbreeding and drift. The classical approach to estimate $N_{e}$ from genetic data involves grouping sampled individuals into discretely defined subpopulations assumed to be panmictic. Importantly, this assumption does not capture the continuous nature of populations genetically isolated by distance. Alternative approaches based on Wright's genetic neighborhood concept quantify the local number of breeding individuals (NS) in a continuous population (as opposed to the global $N_{e}$ ). However, they do not reflect the potential for $N S$ to vary spatially nor do they account for the resistance of a heterogeneous landscape to gene flow (isolation by resistance). Here, we describe an application of Wright's neighborhood concept that provides spatially-explicit estimates of local NS from genetic data in continuous populations isolated by distance or resistance. We delineated local neighborhoods surrounding each sampled individual based on sigma $(\sigma)$, a measure of the local extent of breeding. When $\sigma$ was known, the linkage disequilibrium method applied to local neighborhoods produced unbiased estimates of $N S$ that were highly variable across the landscape. NS near the periphery or areas surrounded by high resistance was as much as an order of magnitude lower compared to the center, raising the potential for a spatial component to extinction vortex dynamics in continuous populations. When $\sigma$ is not known, it may be estimated from genetic data, but two methods we evaluated identified analysis extents that produced considerable bias or error in the estimate of NS. When $\sigma$ is known or accurately estimated, and the assumptions of Wright's neighborhood are met, the method we describe provides spatially explicit information regarding short-term genetic processes that may inform conservation genetic analyses and management.
\end{abstract}

Keywords: dispersal, isolation by distance, isolation by resistance, neighborhood size, $N_{e}, N S$, sGD

\section{INTRODUCTION}

Populations lose allelic diversity and heterozygosity through the processes of genetic drift and inbreeding, respectively (Amos and Harwood, 1998). If this diversity is not replaced by the processes of mutation and immigration, the population loses alleles and heterozygosity at a rate proportional to the number of breeding individuals (Wright, 1931). Diminished genetic diversity may reduce a population's capacity for adaptation in dynamic environments (though dominance, epistasis, and pleiotropy may strongly affect this relationship; Reed and Frankham, 2001), lower the frequency of heterozygotes (and therefore the prevalence of heterozygote vigor or heterosis), favor inbreeding depression, and ultimately increase the risk of extinction (Frankham et al., 2002).

Wright (1931) first defined the concept of effective population size $\left(N_{e}\right)$ as the number of breeding individuals in an ideal population experiencing the same rate of drift as the real population of interest. Factors like unequal sex ratios, a fluctuating population size, overlapping generations, high variability in the distribution of offspring, and non-random mating make the effective size of a population lower than the census size $\left(N_{c}\right)$ because they influence the number of individuals that contribute their genetic variation to the next generation (Frankham et al., 2002). Therefore, the risk of extinction posed by reduced genetic diversity is more closely related to $N_{e}$ than $N_{c}$, making $N_{e}$ is an important parameter in conservation genetics (Lande and Barrowclough, 1987).

$N_{e}$ may be calculated from demographic data (Caballero, 1994; Wang and Caballero, 1999) that are often difficult to acquire from wild populations. Instead, $N_{e}$ is more commonly estimated from genetic data. Long-term genetic estimates of $N_{e}$ may be obtained from the relationship between genetic diversity $(\theta)$ and the mutation rate $(\mu)$ :

$$
\theta=4 N_{e} \mu
$$

Alternatively, short-term genetic estimates of $N_{e}$ may be derived from genetic data sampled from one or more recent generations. Single-sample methods reflect the effective number of breeding individuals $\left(N_{b}\right)$ in the sampled generation, and have been based on rates of drift or inbreeding from heterozygote excess (Pudovkin et al., 1996), linkage disequilibrium (Hill, 1981), and 
molecular co-ancestry (Wang, 2009). A two-sample temporal method (Waples, 1989) estimates the harmonic mean of $N_{e}$ from genetic drift that occurs over the time between samples, but when sampling intervals are from recent generations, it is also a contemporary measure of $N_{e}$. These contemporary estimates of $N_{e}$ differ from Wright's original concept of $N_{e}$, which reflects the long-term harmonic mean number of effective breeding individuals over many generations.

Wright's original concept of $N_{e}$ assumes a closed panmictic population. In many wild populations, however, individuals are continuously distributed across a landscape, and mating probabilities are a function of Euclidean distance (i.e., isolation by distance or IBD; Wright, 1943) or effective distance given the variable resistance of the landscape to gene flow (i.e., isolation by resistance or IBR; McRae, 2006). Such populations become internally structured into continuously overlapping local neighborhoods of breeding individuals. To quantify $N_{e}$ in continuous populations isolated by distance, Wright proposed the concept of a genetic neighborhood (Wright, 1946), defined as

$$
N S=4 \pi \sigma^{2} D
$$

where NS is the number of breeding individuals in the local neighborhood, $\sigma$ is the mean squared parent-offspring dispersal distance along one axis in a two-dimensional habitat (assuming a Gaussian dispersal function), and $D$ is the ideal population density (i.e., the number of ideal individuals per unit area). A genetic neighborhood under isolation by distance forms a circle with a radius of $2 \sigma$, reflecting the local area within which gene flow is high relative to drift (i.e., the neighborhood, rather than the global population, approaches panmixia).

Maruyama (1972) demonstrated that if the local extent of breeding is small relative to the global population, $\sigma^{2} D<1$ and genetic diversity is lost from the global population at a rate approximated by $\sigma^{2} D /(2 N)$ per generation. Conversely, when the local extent of breeding is large and approaches the global population, $\sigma^{2} D>1$ and genetic diversity declines at approximately the same rate as a panmictic population (i.e., $1 / 2 \mathrm{~N}$ per generation). Thus, in continuous populations highly structured by distance or patterns of resistance, the capacity to retain genetic diversity is driven by the local NS rather than the global effective size.

Methods have been developed to infer NS based on the approximately linear relationship expected between the logarithm of spatial distance and genetic distances among individuals (e.g., Rousset, 2000; Hardy and Vekemans, 2002; Rousset and Leblois, 2011). For diploid individuals in a two-dimensional habitat, NS is equal to 1 divided by the slope of the regression between individual genetic distance and the log of distance (Rousset, 2000). Importantly, estimating NS using this approach, or from demographic estimates of $\sigma^{2} D$, provides a single estimate of the local $N S$ for the entire population, assuming no spatial variation in NS.

In the center of a uniformly distributed population isolated by distance, effective population density and neighborhood area are constant, and there is no expectation for spatial variation in NS. However, for genetic neighborhoods near the population's periphery, the edge would be expected to reduce the neighborhood's area, resulting in a lower NS, and giving rise to spatial heterogeneity in NS. Similarly, spatial variation in effective population density $(D)$ would also be expected to produce spatial variation in NS. Furthermore, in populations inhabiting heterogeneous landscapes (i.e., isolation by resistance), patterns of resistance may create variability in the area of genetic neighborhoods and thereby produce spatial variation in NS.

To date, attempts to quantify NS in continuous populations have not addressed the potential for spatial variation in NS. Yet spatial genetic variation in genetic processes is an inherent property of populations isolated by distance or resistance (Rohlf and Schnell, 1971; Ibrahim et al., 1996; Miller, 2005; Shirk and Cushman, 2011). Failure to detect this variation could lead to incorrect inferences regarding the true range of NS across a study area and potentially misinform conservation efforts, particularly near the periphery or in areas where patterns of resistance or population density differ strongly from the rest of the population. Indeed, all wild populations have edges, and they are often of particular interest in conservation genetics (García-Ramos and Kirkpatrick, 1997). A means of detecting spatial variation in NS that provides unbiased estimates even near a population's periphery is needed.

Detecting spatial variation in NS for populations isolated by resistance or distance requires a means to produce estimates of NS across the population at multiple locations or as a continuous surface while accounting for the continuous nature of the population. We previously described an approach (Shirk and Cushman, 2011) to estimate genetic diversity indices spatially using Wright's concept of a genetic neighborhood. The outer extent of Wright's neighborhood in a continuous population isolated by distance is a circle with a radius of $2 \sigma$. By placing this circular neighborhood around each sampled individual, and estimating genetic diversity indices from all individuals within the circle, we matched the sampling unit to the local breeding extent. This produced a spatially explicit estimate of genetic diversity that was not biased by a Wahlund effect (Wahlund, 1928) that occurs when calculating genetic indices at broad extents relative to the local population structure. These analysis extents overlapped in space such that an individual could belong to multiple neighborhoods, reflecting the continuous nature of the population.

In addition to offering a spatially explicit and unbiased means of estimating genetic diversity indices, in Shirk and Cushman (2011), we extended Wright's neighborhood concept to also include populations fitting the IBR model by defining neighborhoods in terms of effective rather than Euclidean distances (e.g., based on cost-weighted distances given a raster model of landscape resistance to gene flow). With this approach, the neighborhood extent is an irregularly shaped kernel rather than a circle (as is the case in an IBD population neighborhood; Figure 1). Whether the neighborhood is a circle or a kernel, the outer edge is $2 \sigma$ from the center. If $N S$ were estimated locally in analysis neighborhoods centered on each sampled individual in the population, it would provide a means to detect spatial variation in NS in continuous populations isolated by distance or resistance.

Because analysis neighborhoods are defined by a radius of $2 \sigma, \sigma$ is a critical parameter. Yet for most species, mean squared parent-offspring dispersal distances are not known and difficult to estimate from field data. Importantly, Neel et al. (2013) found 


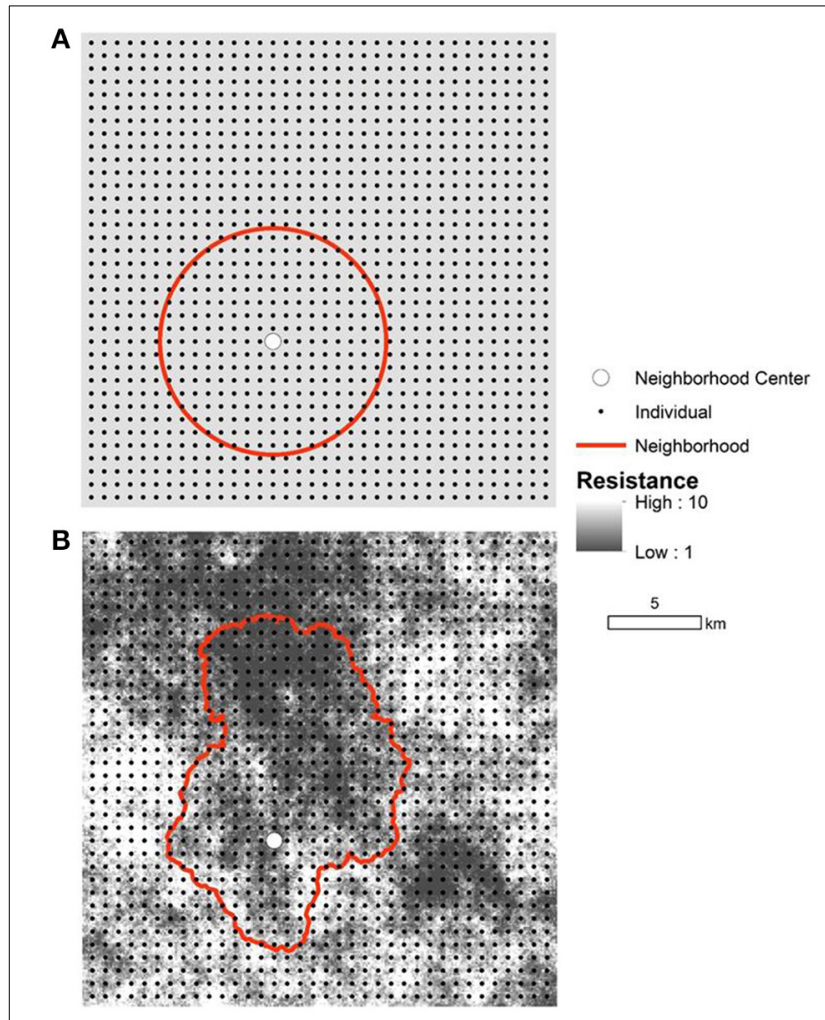

FIGURE 1 | Simulated landscapes, populations, and neighborhoods. The isolation by distance population (IBD; $\mathbf{A}$ ) and the isolation by resistance population (IBR; B) consisted of 1296 individuals (black dot) arrayed in a $36 \times 36$ regular grid. Mating and dispersal probabilities in the simulation were probabilistic functions of the Euclidean distance or effective distance between individuals in the IBD and IBR simulations, respectively. Effective distances were based on cost-weighted distances given a raster model of resistance to gene flow (example in $\mathbf{B}$ ). Resistance varied from 1 (black) to 10 (white) and the spatial pattern differed between replicate IBR simulations. The square landscapes measured $25.6 \mathrm{~km}$ per side. An example of the extent of Wright's genetic neighborhood is shown in each panel (red line) for a single individual at the center (large white circle).

strong bias in the estimate of NS based on the linkage disequilibrium (LD) method when the sampling extent over which $N S$ was calculated did not match the local breeding extent $(2 \sigma)$. Furthermore, they reasoned that a positive or negative inbreeding coefficient $\left(F_{I S}\right)$ would be apparent if the sampling extent was larger or smaller than the extent of local breeding, respectively. Iteratively exploring a range of neighborhood extents until $F_{I S}$ approached zero could therefore be used to identify Wright's neighborhood extent $(2 \sigma)$. If this or other approaches based on genetic data were to reliably identify the appropriate analysis extent, it would allow for unbiased estimates of NS in continuous populations without requiring the difficult task of inferring $\sigma$ from field data.

In this study, we used an approach analogous to Shirk and Cushman (2011) to assess spatial variation in NS in both simulated IBD and IBR populations and a real population of mountain goats (Oreamnos americanus) inhabiting a complex landscape. Based on these simulations, we explored several questions. First, we evaluated the ability of our spatially-explicit implementation of Wright's neighborhood concept to accurately estimate NS in populations isolated by distance or resistance without bias. Second, we evaluated two approaches to inferring $\sigma$ from the same genetic data used to estimate NS. Third, we examined whether proximity to the population edge produced bias in the estimate of NS. Lastly, we explored spatial variation in NS in the simulated IBD and IBR populations as a real population of mountain goats. We expected estimates of NS to be unbiased as long as the radius of the neighborhood was equal to $2 \sigma$. We also expected strong spatial variation in NS within the IBD, IBR, and mountain goat populations, and that peripheral areas or regions isolated by strong patterns of resistance would have the lower NS relative to the population core.

\section{MATERIALS AND METHODS SIMULATED POPULATIONS, LANDSCAPES, AND DISTANCES}

We simulated mating and dispersal in continuous populations isolated by distance (IBD) or resistance (IBR). Each simulation was replicated 100 times. In all simulations, the landscape was represented by a square $256 \times 256$ cell raster grid with square cells measuring $100 \mathrm{~m}$ per side. The populations were constant in size $(N=1296)$ and distribution (a $36 \times 36$ uniform square grid with $700 \mathrm{~m}$ between individuals), however, the IBD and IBR landscapes differed in their capacity to limit the movement of mating and dispersing individuals. In the IBD population, mating and dispersal probabilities were based on the Euclidean distance between individuals. Because the population size and distribution was constant in all simulations, the Euclidean distance between individuals was constant in the IBD populations. In the IBR populations, however, mating and dispersal probabilities were a function of the Euclidean distance weighted by the landscape's resistance to gene flow (i.e., the effective or cost-weighted distance).

We used the program QRULE (Gardner, 1999) to create the IBR landscapes, with resistance values ranging from 1 to 10 in equal area proportions. These values represented resistance to movement such that the "cost" to traverse a grid cell was equal to the cell size $(100 \mathrm{~m})$ times the resistance. Thus, a value of one implies that the Euclidean and effective distances are equal. A population isolated by distance can be thought of as inhabiting a raster landscape with a resistance of one in all cells. Values greater than one indicate additional cost to movement beyond the effect of distance alone (e.g., suboptimal habitat types). Resistance values were spatially distributed with a QRULE $H$ parameter value of 0.1 , which produced clumped distributions that are commonly observed in real landscapes (Figure 1). Because this pattern of resistance varied between replicate runs, the effective distance between individuals varied among the IBR simulations.

Mating and dispersal probabilities in the simulations were based on the Euclidean (for the IBD population) or effective (for the IBR population) distances between individuals. We calculated pairwise Euclidean distances in an $N \times N$ matrix $(N=$ 1296) using the Ecodist package (Goslee and Urban, 2007) in R (R Development Core Team, 2013). We calculated effective distances with the gdistance package (van Etten, 2011) in R based on the cumulative cost of the least-cost path given the 
raster model of landscape resistance for each IBR simulation replicate.

\section{POPULATION GENETIC SIMULATIONS}

We used the population genetic simulation software CDPOP (Landguth and Cushman, 2010) to simulate mating and dispersal in IBD and IBR populations (100 replicates each). CDPOP is an individual-based simulator of population genetic processes. It simulates mating and dispersal in a finite population assigned to fixed locations. Over a user-specified number of generations, CDPOP records allele usage by all individuals per generation, and computes various population genetic statistics. In each generation, adult individuals mate according to a user-specified mating system and probability function based on proximity in Euclidean or effective distance. Once mated, females give birth to a number of offspring determined by a user-specified probability function which can also control the sex ratio at birth. After birth, adult mortality occurs probabilistically based on user-specified demographic information. Finally, vacant locations where adults have died are filled by dispersing offspring. Dispersal probabilities follow a user-specified function based on Euclidean or effective distances to the vacant locations. If all locations are occupied, any remaining offspring not yet assigned to a location are eliminated. Unoccupied sites may be filled by immigrants or left open.

We parameterized the IBD and IBR CDPOP simulations to reflect a hermaphroditic animal distributed at a moderate uniform density with limited movement capability relative to the size of the landscape, thus allowing for spatial patterns in effective population size to emerge over time. Parameters matched the assumptions of an ideal Wright-Fisher population and Wright's genetic neighborhood. Thus, the census number of individuals within a genetic neighborhood was equal to effective number of breeding individuals, allowing for genetic estimates of NS to be related to the true NS. We specified mating and dispersal probabilities to be a Gaussian function of Euclidean or effective distance (for the IBD and IBR populations, respectively), with a maximum equal to $30 \%$ of the landscape width (7680 $\mathrm{m}$ in Euclidean units). Except for varying mating and dispersal probability as a function of the landscape, the CDPOP parameters were the same in all simulations. Generations were discrete and non-overlapping. There was no selection, mutation, or immigration from outside the population. Individuals were diploid. Mating was sexual (equivalent to hermaphroditic with selfing not allowed). The number of offspring was based on a Poisson distribution with a mean of 4 , providing ample offspring to fill all vacant locations. The simulation tracked alleles at 20 loci, with 20 alleles randomly assigned per locus to the first generation. At the end of 300 generations, CDPOP recorded the genotypes of all individuals in the population.

\section{EMPIRICAL LANDSCAPE AND GENETIC DATA}

To explore spatial variation in the NS of a real population, we used genetic data previously collected (Shirk et al., 2010) from mountain goats in the Cascade Range in Washington State (USA). The majority of the area is encompassed by national forests and national parks. The landscape is mountainous and covered with montane forests, except at elevations above about $1400 \mathrm{~m}$, where subalpine parkland, rocky alpine summits, and glaciers predominate. Elevation varies from near sea level to almost $4400 \mathrm{~m}$. One major interstate highway, several state highways, and a network of logging roads extend throughout the study area.

We previously genotyped 135 mountain goats sampled from this region at 18 microsatellite loci (Shirk et al., 2010). These genotypes were used to calculate pairwise inter-individual genetic distances, which we related to effective distances (based on various resistance hypotheses) in a causal modeling framework (Cushman et al., 2006; Shirk et al., 2010). This identified a model of landscape resistance as causal relative to alternative resistance models or null models of isolation by distance or barriers. The causal model accounted for the influence of elevation, land cover, and roads on gene flow. In the present analysis, we used this resistance model to define genetic neighborhoods for mountain goats within the study area, and the genotypes to estimate NS within the neighborhoods.

\section{INFERRING $\sigma$ FROM GENETIC DATA}

We evaluated two approaches to using genetic data to infer the mean squared parent-offspring dispersal distance $(\sigma)$ that determines the spatial extent of Wright's neighborhood. We used the program sGD (Shirk and Cushman, 2011) to calculate the inbreeding coefficient $\left(F_{I S}\right)$ and a chi-square test for goodness-offit to Hardy-Weinberg equilibrium (HWE) within genetic neighborhoods centered on each individual's location in the IBD and IBR simulated populations. The outer extent of each neighborhood was defined in terms of $\sigma$, which was recorded by CDPOP during the simulations. Because of the Wahlund effect that occurs when the analysis extent does not match Wright's genetic neighborhood (Neel et al., 2013), we expected $F_{I S}$ to be positive and the HWE test $p$-value to be significant ( $\alpha=0.05$; the null hypothesis was that the sample was in HWE) when the analysis extent was > $2 \sigma$. Conversely, when the analysis extent was $<2 \sigma$, we expected $F_{I S}$ to be negative and the HWE test $p$-value to not be significant. To quantify bias and error when $\sigma$ was not known, we estimated $\sigma$ by iteratively increasing the analysis neighborhood from 5 to $50 \%$ of the Euclidean or effective distance (for the IBD and IBR simulations, respectively) to cross the landscape, in $5 \%$ increments. The estimated $\sigma$ was defined by the distance at which $F_{I S}$ was closest to zero or the HWE test $p$-value was closest to 0.05 .

\section{ESTIMATING NS}

To estimate NS spatially, we modified the previously described program sGD (Shirk and Cushman, 2011), which calculates genetic diversity indices within genetic neighborhoods given a spatially referenced set of co-dominant input genotypes and a matrix of pairwise inter-individual landscape distances. Distances may be calculated in Euclidean or effective units as appropriate for the landscape and population of interest. For each genotyped individual in the sample, sGD uses the distance matrix to identify all other individuals that are below a user specified threshold distance (i.e., the neighborhood radius). Genetic diversity indices are then calculated from the genotypes of all individuals within each neighborhood. For this analysis, we modified sGD to also estimate $N S$ within the same genetic neighborhoods via a dependency on the program NeEstimator (Do et al., 2014). NeEstimator 
implements the most widely used one-sample estimator, the Burrow's method based on linkage disequilibrium (LD) resulting from genetic drift in a finite population (Cockerham and Weir, 1977; Hill, 1981; Weir, 1996). The LD method estimates the number of breeding individuals responsible for the sample $\left(N_{b}\right)$, for the parental generation (Hill, 1981; Waples, 2006; Waples and Do, 2008). The method to estimate NS spatially (as well as the original methods to estimate common indices of genetic diversity) is now implemented in the sGD package in $\mathrm{R}$ ( $\mathrm{R}$ Development Core Team, 2013).

For each replicate in the IBD and IBR simulations, we used the sGD package to estimate NS within genetic neighborhoods centered on each of the 1296 individuals in the population. The radius of the neighborhood was determined by the mean squared parent-offspring dispersal distance $(\sigma)$, which was recorded by CDPOP during the simulations. We varied this extent from 1 to $4 \sigma$, using Euclidean distances for the IBD populations and effective distances for the IBR populations (calculated as described above). These pairwise distances defining the genetic neighborhoods were the same distances that determined mating and dispersal probabilities in the CDPOP simulations. After 300 simulated generations, we used the sGD package to calculate $\widehat{\mathrm{N}}_{b}$ within neighborhoods with at least 20 individuals (to reduce error from small sample sizes). To limit bias due to inclusion of rare alleles, we included only alleles with a frequency $>0.01$ (Waples and Do, 2010). Because our simulation parameters met the Wright-Fisher assumptions of an ideal population, the number of individuals in each genetic neighborhood of radius $2 \sigma$ was equal to the NS based on Wright's definition. This provided a means to compare $\widehat{\mathrm{N}}_{b}$ to the true NS. We also used the sGD package to estimate NS spatially from the mountain goat genotypes, resistance model, and estimate of $\sigma$ described above and published in Shirk et al. (2010).

\section{RESULTS}

In the CDPOP simulations, the lack of mutation and immigration allowed drift and inbreeding to reduce allelic diversity and heterozygosity in the population over time (Figure 2). Observed heterozygosity $\left(H_{o}\right)$ was lower than expected heterozygosity $\left(H_{e}\right)$, resulting in a positive inbreeding coefficient $\left(F_{I S}\right)$. This reflects a Wahlund effect due to substructure within the sampling extent (i.e., the entire population). The population was more highly structured in the IBR simulation compared to the IBD simulation, and inbreeding was correspondingly higher. That inbreeding plateaued after 20-30 generations indicates population genetic structure formed very rapidly, and was stable for the remainder of the simulation.

In the IBD simulations, mean $\sigma$ across 100 replicates was $3058 \mathrm{~m}$ (SD $82 \mathrm{~m}$ ) in Euclidean distance units. In the IBR simulations, mean $\sigma$ was $10950 \mathrm{~m}$ (SD $650 \mathrm{~m}$ ) in effective distance units. As the analysis neighborhood radius was increased from 1 to $4 \sigma$, the number of individuals in the neighborhood $(N)$ and the estimate of $N S\left(\hat{N}_{b}\right)$ increased (Figures $\mathbf{3 A}, \mathbf{B}$, respectively). In both the IBD and IBR simulations, the mean $\hat{N}_{b}: N S$ ratio for all neighborhoods approached 1 only when the analysis extent radius was $2 \sigma$ (Figure $3 \mathrm{C}$ ). Thus, $\hat{N}_{b}$ was not biased when the analysis extent matched the extent of Wright's genetic neighborhood. However, at analysis extents $<2 \sigma, \hat{N}_{b}$ :NS was $>1$, indicating
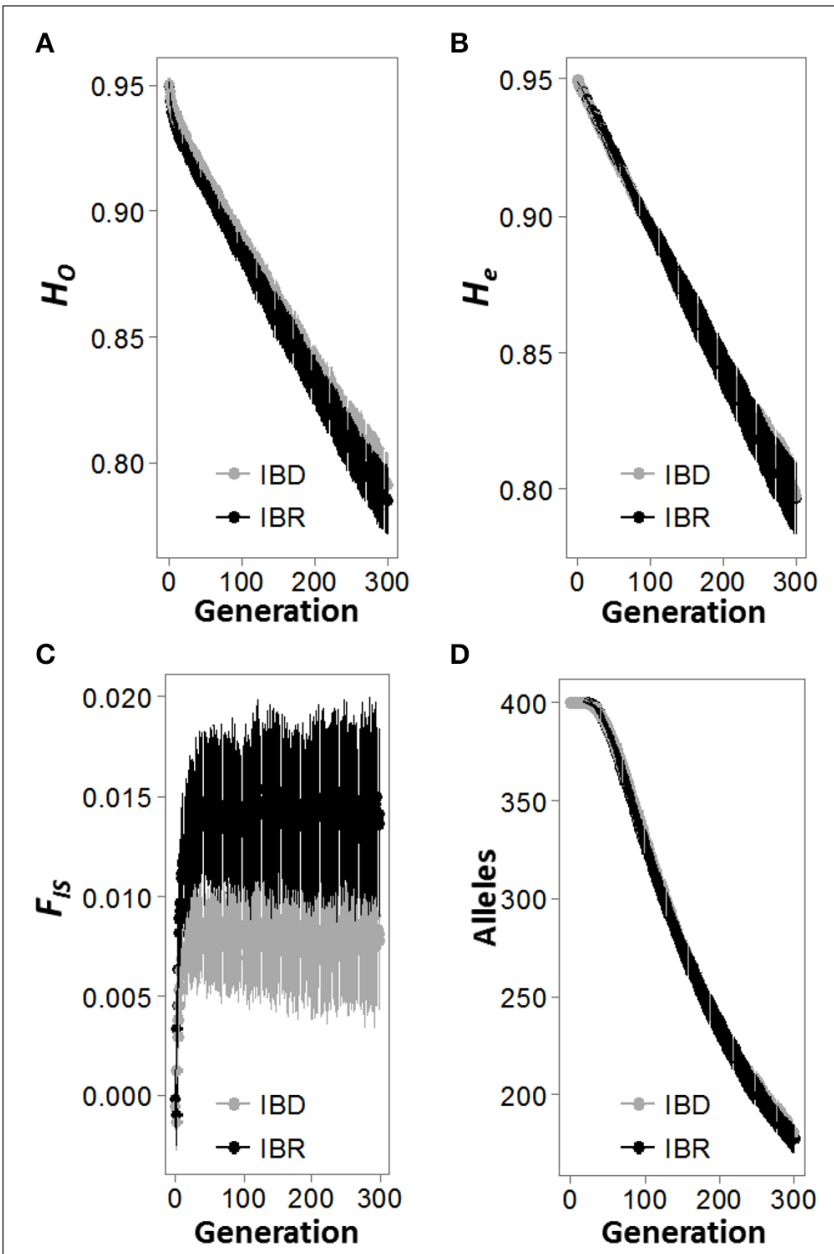

FIGURE 2 | Change in population genetic indices over $\mathbf{3 0 0}$ simulated generations. Observed heterozygosity $\left(H_{0} ; \mathbf{A}\right)$, expected heterozygosity $\left(H_{e} ; \mathbf{B}\right)$, inbreeding coefficient $\left(F_{I S} ; \mathbf{C}\right)$, and the number of alleles $(A ; \mathbf{D})$ in the population were recorded for each generation, for both the isolation by distance (IBD; gray line) and isolation by resistance (IBR; black line) simulations. Each plot represents the mean and standard deviation of 100 replicate simulations.

bias toward overestimation. Conversely, at analysis extents $>2 \sigma$, $\hat{N}_{b}: N S$ was $<1$, indicating a bias toward underestimation.

As the analysis neighborhood increased in radius from 1 to $4 \sigma$, the mean $F_{I S}$ within neighborhoods in the IBD and IBR populations transitioned from negative to positive values at $2 \sigma$, though the variance across replicate runs was high (Figure 4A). Similarly, a chi-square test for HWE within each neighborhood became significant at $\alpha=0.05$ when the analysis neighborhood approached $2 \sigma$, though we observed high variability across replicate runs (Figure 4B). Using the above relationships to infer $\sigma$ by iteratively increasing the analysis neighborhood, we found the point at which the HWE test $p$-value became significant was often less than $\sigma$. Estimating NS using this biased estimate of $\sigma$ to define the analysis neighborhoods added considerable error and a strong bias toward overestimating NS, in both the IBD and IBR populations (Table 1). The distance at which $F_{I S}$ transitions from negative to positive produced highly variable estimates of $\sigma$, which 

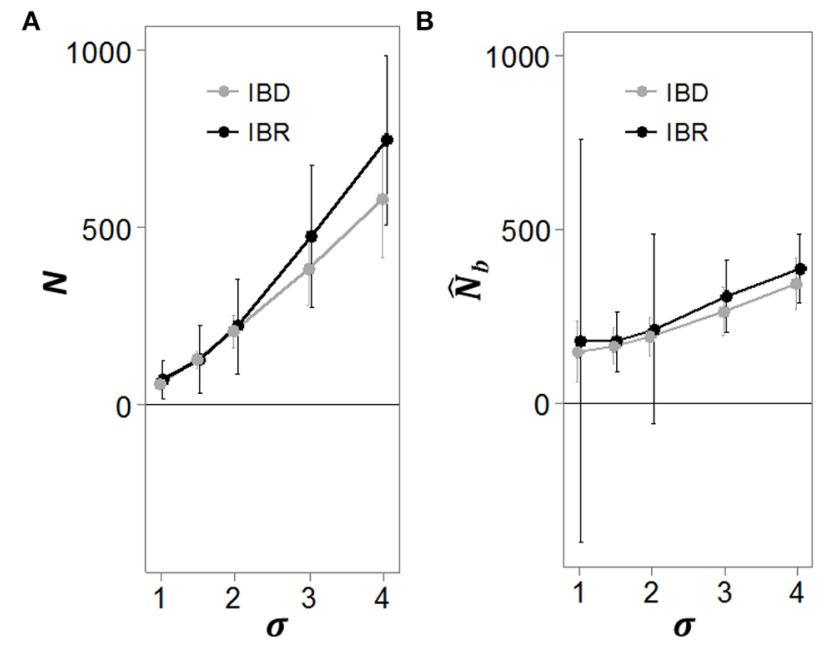

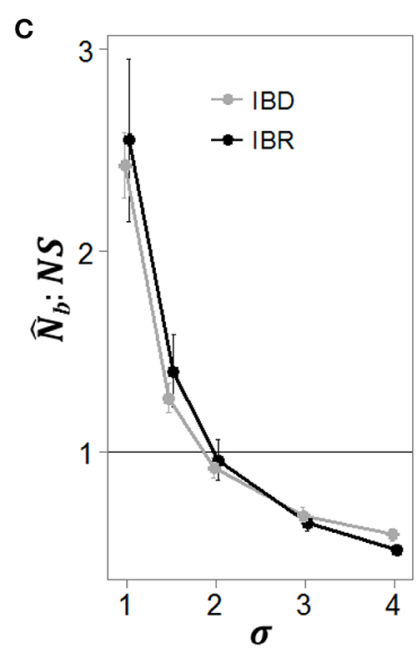

FIGURE 3 | Bias in the estimate of $N S$ as a function of the analysis window extent. Simulated genotypes at generation 300 were grouped into overlapping analysis neighborhoods of varying extents. The analysis extents were defined in terms of $\sigma$, the mean squared parent-offspring dispersal distance recorded during the simulations. In (A), the number of individuals per neighborhood at each analysis extent is shown for both the isolation by distance (IBD; gray line) and isolation by resistance (IBR) simulations. In (B), $\hat{N}_{b}$ (the estimated number of breeding individuals responsible for the sample) calculated using the LD method (implemented in the program NeEstimator) at varying analysis neighborhood extents is shown. We used $\hat{N}_{b}$ as an estimate of Wright's neighborhood size (NS; the actual number of breeding individuals in a neighborhood with a radius of $2 \sigma$ ). Bias in this estimate at analysis neighborhood extents that do not match the extent of local breeding becomes apparent when the ratio of $\hat{N}_{b}: N S$ is $<$ or $>1.0$ (C). Each plot represents the mean and standard deviation of 100 replicated simulations.
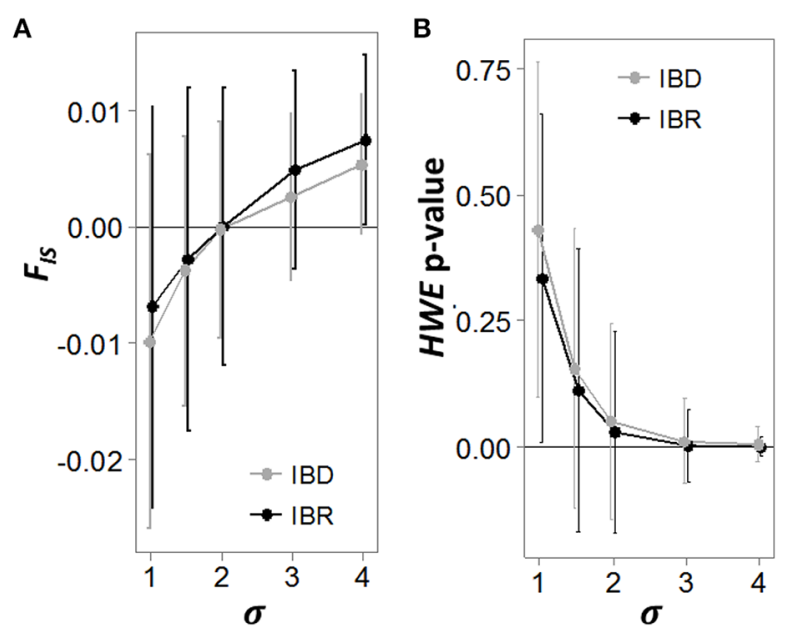

FIGURE $4 \mid F_{I S}$ and HWE test $p$-value as a function of the analysis window extent. Simulated genotypes after 300 generations were grouped into overlapping analysis neighborhoods of varying extents. The analysis extents were defined in terms of $\sigma$, the mean squared parent-offspring dispersal distance recorded during the simulations. The inbreeding coefficient $\left(F_{/ S} ; \mathbf{A}\right)$ and the $p$-value for a chi-square test of Hardy-Weinberg equilibrium (HWE; B) were calculated for each analysis neighborhood extent in both the isolation by distance (IBD; gray lines) and isolation by resistance (IBR; black lines) simulations. Each plot represents the mean and standard deviation of 100 replicated simulations.

when used as the analysis neighborhood also added considerable error to the estimate of NS, but no strong bias (Table 1).

When the analysis extent matched the extent of breeding at a radius of $2 \sigma$, the distribution of $\hat{N}_{b}$ :NS ratios was approximately
Table 1 | Mean ratio of $\widehat{N}_{b}$ (the estimated number of breeding individuals in the neighborhood sample) to NS (the true neighborhood size), and mean absolute error (MAE; as a percent of the true neighborhood size) in the estimate of NS, over 100 replicate simulations.

\begin{tabular}{lccr}
\hline Method & Landscape & Mean $\widehat{\boldsymbol{N}}_{\boldsymbol{b}}: \boldsymbol{N S}$ ratio & MAE(\%) \\
\hline$F_{/ S}$ & IBD & 1.05 & 28.5 \\
HWE & IBD & 1.21 & 23.9 \\
Known $\sigma$ & IBD & 0.94 & 7.6 \\
$F_{/ S}$ & IBR & 1.04 & 32.4 \\
HWE & IBR & 1.30 & 25.4 \\
Known $\sigma$ & IBR & 0.96 & 9.1 \\
\hline
\end{tabular}

An unbiased estimate of NS without error would have a ratio of 1.0 and a MAE of 0 . Values are given for the inbreeding coefficient $\left(F_{I S}\right)$ and Hardy-Weinberg equilibrium (HWE) methods of inferring sigma $(\sigma)$ and when $\sigma$ is known in both the isolation by distance (IBD) and isolation by resistance (IBR) simulated landscapes.

centered on 1 in neighborhoods at varying distances from the edge, in both the IBD and IBR populations (Figure 5), indicating no bias in the estimate of NS due to edge effects. Furthermore, spatial variation in NS was highly apparent in all simulations. In the IBD simulations, the mean NS across all simulations was highest in the center of the landscape and decreased uniformly toward the periphery (Figure 6A). In the IBR simulations, NS also tended to be higher in the center of the landscape relative to the periphery (Figure 6B). However, the complex pattern of resistance created complex patterns in NS that varied between each simulated IBR landscape. Spatial variation in NS was also 


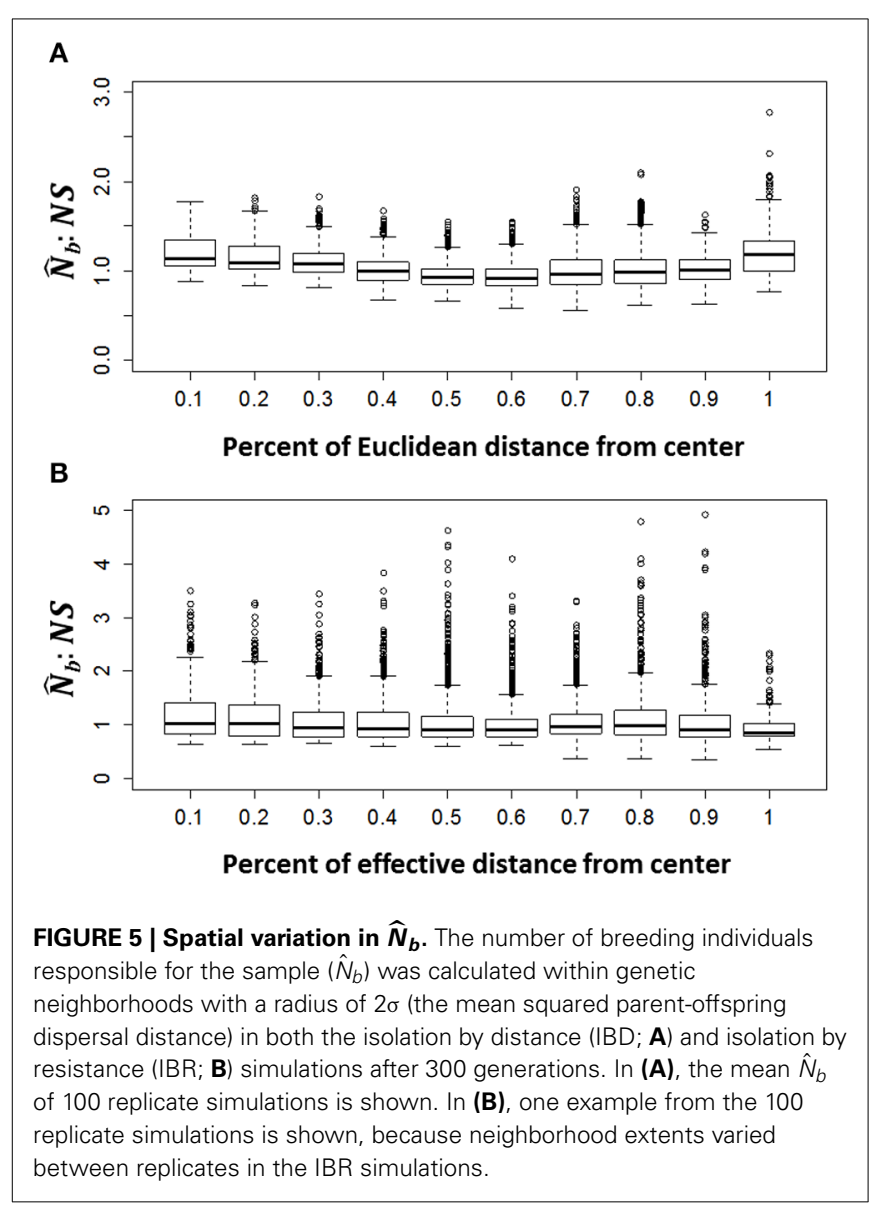

strong in a population of mountain goats in the Cascade Range, Washington, USA (Figure 7). The southern part of the population, which has a high local density of individuals, had the largest relative NS whereas in the north, where mountain goats are sparsely distributed, NS was comparatively low.

\section{DISCUSSION}

Spatial genetic variation is an expected property of continuous populations when the extent of the population exceeds the local extent of dispersal and mating (Ibrahim et al., 1996; Miller, 2005; Shirk and Cushman, 2011). In this study, we describe an application of Wright's neighborhood concept designed to quantify spatial variation in NS for continuous populations isolated by distance or resistance. We previously applied this method to estimating spatially-explicit indices of genetic diversity in Shirk and Cushman (2011). Here, we extend this approach to also estimate $N S$ in local analysis neighborhoods surrounding each sample location. Matching the analysis neighborhood to the local extent of breeding in the population provided unbiased estimates even near the periphery of a population, and revealed complex patterns of NS that have important conservation implications for continuous populations.

Neel et al. (2013) applied a similar approach to estimate $N S$ in local neighborhoods, but not in a spatially-explicit manner and only for populations isolated by distance. There were also differences between these studies in terms of how genetic

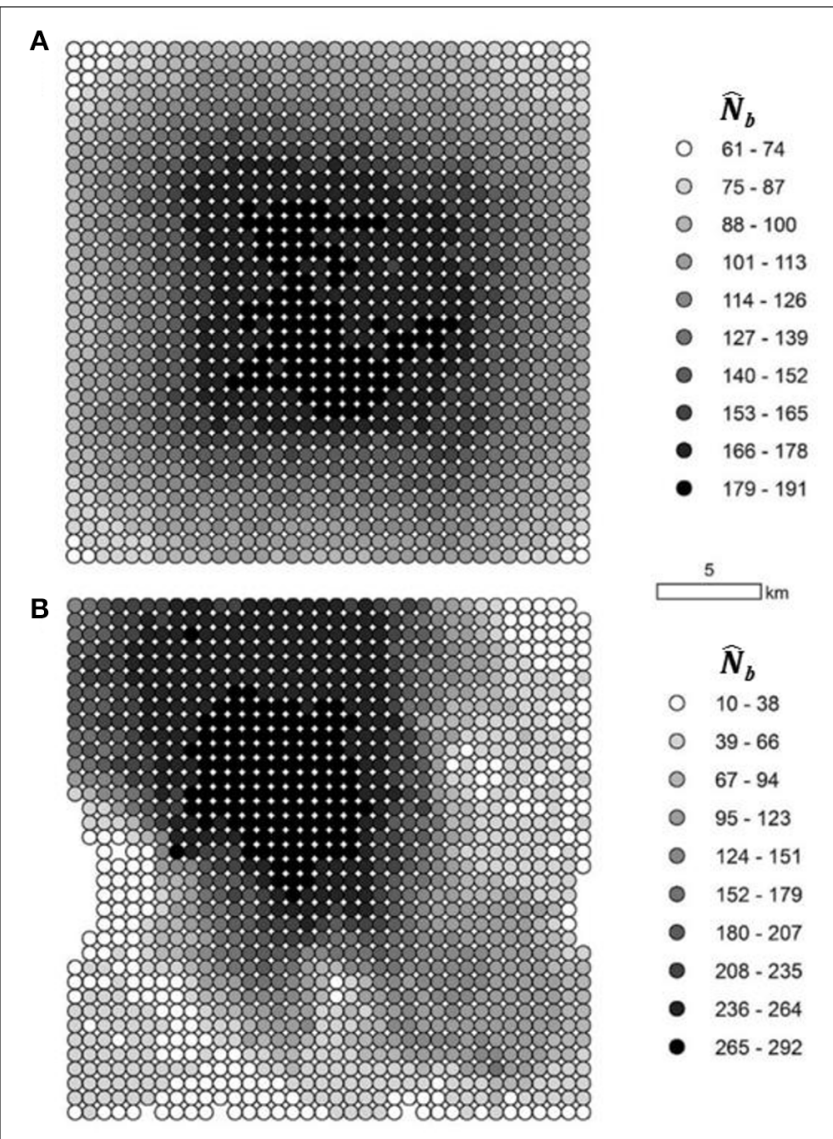

FIGURE 6 | Edge bias in $\widehat{\boldsymbol{N}}_{\boldsymbol{b}}$. Simulated genotypes after 300 generations were grouped into overlapping analysis neighborhoods with a radius of twice the mean squared parent-offspring dispersal distance $(\sigma)$ recorded during the simulations, for both the isolation by distance (IBD; $\mathbf{A}$ ) and isolation by resistance (IBR; B) populations. $\hat{N}_{b}$ (the estimated number of breeding individuals responsible for the sample) was calculated for each neighborhood using the LD method (implemented in the program NeEstimator). We used $\hat{N}_{b}$ as an estimate of Wright's neighborhood size (NS; the actual number of breeding individuals in a neighborhood with a radius of 2r). Bias in the estimate of $N S$ at varying percent distances from the center of the population would be evident if the ratio of $\hat{N}_{b}: N S$ is $<$ or $>$ 1.0 (C). The dark horizontal line in each boxplot represents in median ratio of all neigborhodos and the top and bottom of the box depict the 25th and 75th percentile of ratios. The whiskers extend to the smallest and largest values in the data that are not outliers (open circles). Each plot summarizes 100 replicate simulations.

neighborhoods were defined and simulated. Wright's neighborhood assumes a Gaussian dispersal function and an outer extent that is a circle with a radius of $2 \sigma$. This extent contains about $87 \%$ of the potential parents for an individual at the center. In Neel et al. (2013), the genetic neighborhood was a square containing $100 \%$ of potential parents and the distances between parents and offspring followed a uniform distribution. In the present analysis, our simulations were based on a Gaussian dispersal function and our estimate of NS was based on a neighborhood extent $2 \sigma$ from the center, thus matching Wright's definition.

Despite these differences, both our study and Neel et al. (2013) identified the same bias in IBD populations when the analysis 

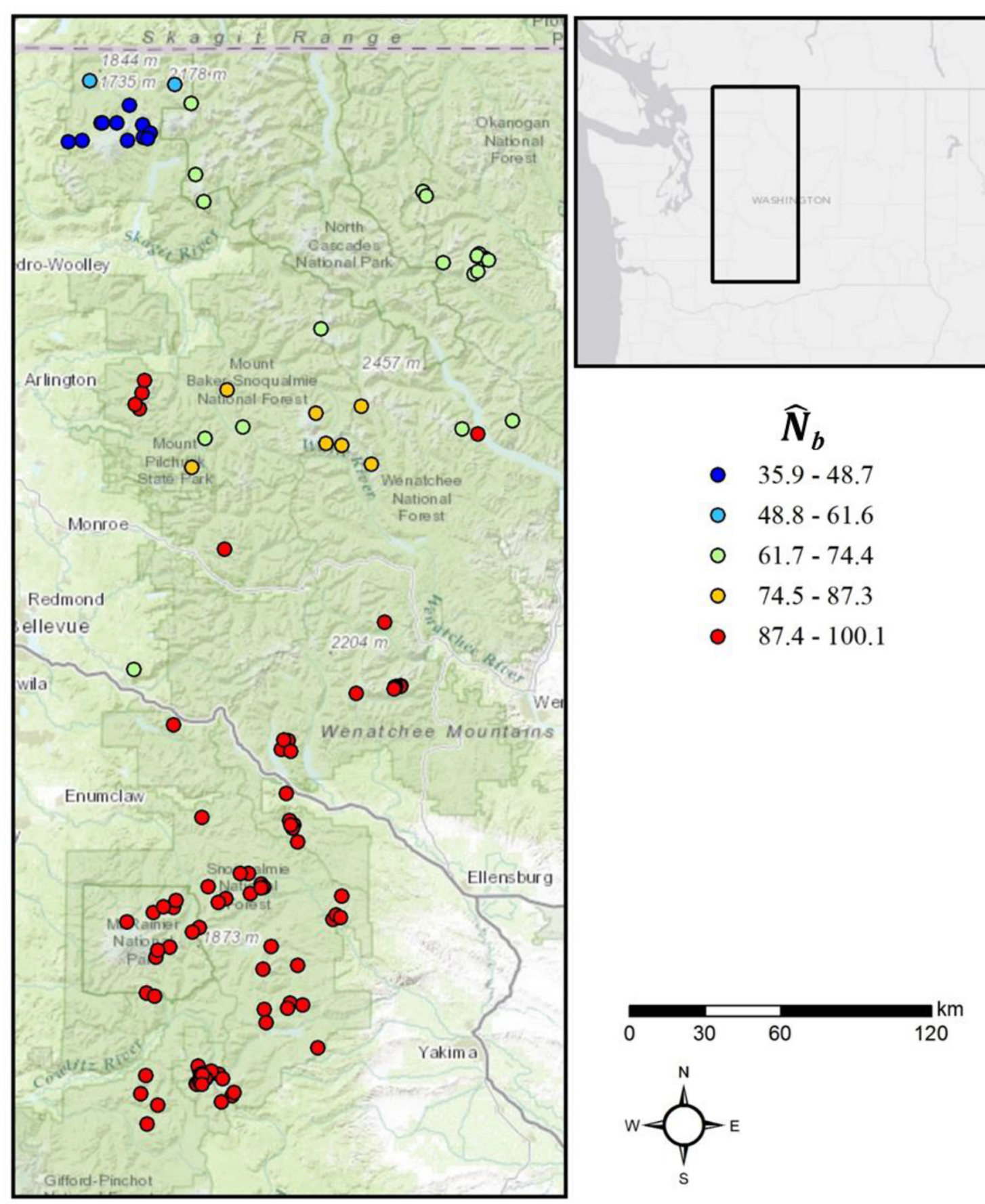

FIGURE 7| Spatial variation in mountain goat $\widehat{\boldsymbol{N}}_{\boldsymbol{b}}$. Genotypes sampled from a population of mountain goats inhabiting the Cascade Range, Washington, USA were grouped into overlapping neighborhoods based on effective distances calculated from an empirical resistance model and a radius defined by twice the estimated mean squared parent-offspring dispersal distance $(\sigma)$. The estimated number of breeding individuals responsible for the sample $\left(\hat{N}_{b}\right)$ was calculated for each neighborhood using the LD method (implemented in the program NeEstimator). Warm colors indicate higher relative $\hat{N}_{b}$ and cool colors represent lower relative $\hat{N}_{b}$ values. neighborhood was too large or small compared to Wright's neighborhood. Specifically, when the analysis neighborhood radius was greater than Wright's neighborhood, Neel et al. (2013) observed a downward bias in NS (due to linkage disequilibrium arising from a two-locus Wahlund effect) and positive $F_{I S}$ values (due a single locus Wahlund effect that produced fewer than expected heterozygotes). Conversely, when the analysis neighborhood radius was smaller than Wright's neighborhood, the bias was opposite; NS was overestimated and negative $F_{I S}$ values were observed due to an excess of heterozygotes compared 
to Hardy-Weinberg expectations. Our study confirms this bias under a strict definition of Wright's neighborhood.

We have extended Wright's genetic neighborhood concept to estimate NS in continuous populations isolated by resistance. The key to this approach was to measure landscape distances, including the neighborhood radius $(2 \sigma)$, in terms of effective rather than Euclidean units. Placing a kernel with this effective distance radius over each sampled individual defined an area within which most mating occurs, and is completely analogous to the circular Wright's neighborhood in a population isolated by distance. Indeed, one can think of IBD and IBR as part of the same continuum. IBD is equivalent to a resistance surface where all cells have a resistance of one (i.e., the cost of traversing a cell is equal to one times the Euclidean distance across the cell). In more resistant landscapes, values above one are present, and, for the same neighborhood radius, the added resistance reduces the distance to the outer edge. In this way, complex patterns of resistance owing to landscape heterogeneity create irregularly shaped kernels. However, as long as distance units are weighted to account for resistance in the landscape (i.e., effective distances), in ecological space, the neighborhood is still a circle. That our results were very similar between the IBD and IBR simulations supports the notion that Wright's neighborhood concept is applicable to both forms of continuous population structure.

In most wild populations, individuals mate and disperse over limited distances relative to the extent of the population. For this reason, wild populations generally exhibit some form of IBD or IBR, and are therefore not panmictic. However, many studies overlook this possibility, and instead estimate $N_{e}$ from discrete groups of sampled individuals assumed to be panmictic (e.g., the Aspi et al., 2006 estimate of $N_{e}$ assumes the border of Finland delineates a closed panmictic population of wolves). Neel et al. (2013) and this study suggest assuming panmixia when in fact the population is continuous creates a mismatch between the sampling extent and the extent over which local breeding occurs, resulting in a biased and unreliable estimate that does not reflect $N_{e}$ for the global population nor the local NS. Moreover, the single estimate for an entire sampling area may mask substantial spatial heterogeneity in the local NS. This is troubling because it suggests past studies that have not accounted for the continuous nature of the population under study may have produced biased and spatially inaccurate inferences regarding the true pattern of NS on the landscape, potentially misinforming conservation planning.

The bias and error that result from violating the assumption of a closed, panmictic population within the sampling extent underscores the need to rigorously identify the mode of genetic isolation prior to estimating $N_{e}, N S$, and other population genetic indices. We have already discussed the risk in assuming a discrete panmictic subpopulation structure when the population is actually continuous. However, there is also risk in misconstruing IBD and IBR in a continuous population. Many studies of population genetic structure explore the fit of IBD to the population of interest, and most find a negative relationship between distance and relatedness, at least when the sampling extent is large relative to area of local breeding. However, most populations inhabit heterogeneous landscapes that variably resist movement and gene flow, raising the potential that IBR models outperform the IBD model in reflecting the true pattern of genetic isolation on the landscape. Yet few studies rigorously compare IBD to alternative IBR models. If we were to assume IBD in our analysis of the IBR simulations, the extent of our analysis neighborhoods (a circle) would not have matched the irregular kernel characterizing the true breeding neighborhood, likely leading to bias and increasing error. The converse is also true. Thus, a thorough examination of the mechanism driving population genetic structure is an essential preliminary step. Recent studies have described approaches to compete various models of genetic isolation against each other to infer the most likely to be causal (Cushman et al., 2006, 2013; Shirk et al., 2010), facilitating this task.

An important component of spatial variation in NS is the edge effect that appears at the periphery of a population. If a population is genetically structured, the theoretical expectation is for reduced gene flow, NS, and neutral genetic diversity near the periphery relative to the core (Caughley, 1994; García-Ramos and Kirkpatrick, 1997), though variation in local population densities and resistance could create more complex patterns. This expectation has been empirically observed in both plant and animal populations, though not consistently (Eckert et al., 2008). In the IBD simulation, where the population density was uniform and genetic isolation a constant function of Euclidean distance, this theoretical expectation was clearly observed. An edge effect was also apparent in peripheral areas in the IBR simulation, though the spatial variation in NS also appeared to be influenced by the complex patterns of resistance. Interestingly, the pattern of NS on the landscape in the empirical mountain goat population was dominated by the variable density of individuals in the population (which much greater in the south than the north), rather than the edge proximity. This demonstrates how heterogeneous landscapes and variable population density can create complex spatial patterns of NS (and concomitant spatial variation in management implications) that can only be elucidated with a spatially explicit approach such as the one described here.

Spatial variation in NS apparent in the simulated and empirical mountain goat populations raises the potential for a spatial component to extinction dynamics in continuous populations. For example, NS near the periphery of the simulated IBR population shown in Figure 6B was as low as 10, while in the center it approached 300. Franklin (1980) proposed a minimum effective size of 50 is required to avoid inbreeding depression in the short term, though some reports consider the minimum number to be greater (Reed and Bryant, 2000). In this example, the periphery of the population would be at risk of decline, even though the core might initially remain viable. Eventually, however, the decline of the edge neighborhoods may contract the population inward, lowering NS in areas adjacent to the original periphery, and potentially creating a wave of inbreeding depression that could threaten even the centermost neighborhoods. If this dynamic occurs in wild populations, it suggests maintaining a sufficiently large NS in the periphery is an important conservation target to avoid a greater threat to the entire population.

While the neighborhood approach implemented in the sGD package affords a means to detect spatial variation in NS and thereby provide new information for conservation genetic 
analyses and genetic management of populations, we note several important limitations and assumptions that need to be met for reliable estimates. First, to produce a strong spatial representation of variation in NS requires a large number of sample genotypes distributed over a broad area yet with sufficient local density to supply a sufficient sample size. This is not possible for rare or very difficult to sample species. Because rare species are often the most threatened and of high interest to conservation genetics, this is a major limitation. For this reason, the neighborhood approach implemented in $s G D$ may be most applicable for larger populations or simulations. In the context of conservation genetics, $s G D$ may be particularly useful for predicting the change and spatial variation in NS for simulated continuous populations under scenarios of future landscape change due to disturbance, climate change, or conservation efforts (see Wasserman et al., 2013 for an example).

Another important limitation of the neighborhood approach to estimating NS is the need for an accurate estimate of $\sigma$. This parameter is not known for most wild populations. Inferring $\sigma$ from the same genetic data used to estimate NS would avoid the need to collect additional data. Neel et al. (2013) proposed that if genetic samples were collected broadly, $F_{I S}$ values could be calculated iteratively over a range of analysis window extents, and the transition from negative to positive $F_{I S}$ values might be used to estimate $\sigma$. Our study confirms this expectation in terms of the mean distance at which $F_{I S}$ approached zero, but the variance was high, adding substantial error to the estimate of $\sigma$ and NS. Similarly, a chi-squared test for HWE generally became significant at a neighborhood radius of $2 \sigma$ in our simulations, with somewhat lower error, but was biased toward underestimating $\sigma$ (and therefore overestimating NS). This bias was likely driven by lower sample sizes at smaller analysis extents not having sufficient power to reject the null hypothesis that the samples were in HWE. The magnitude of spatial variation in NS we observed in both the IBD and IBR populations when $\sigma$ was known was much greater than the error and bias introduced when $\sigma$ was estimated using the HWE and $F_{I S}$ methods, suggesting these methods may still provide insight into the relative patterns of NS on the landscape, but more robust methods are clearly needed.

Other alternatives to estimating $\sigma$ have been explored. The autocorrelation in genetic distances as a function of landscape distance has been related to dispersal distances in several studies (Sokal and Wartenberg, 1983; Hardy and Vekemans, 1999; Smouse and Peakall, 1999). In addition, the approximately linear relationship expected between the logarithm of spatial distance and genetic distances among individuals has been used to infer both $\sigma$ and NS (Rousset, 2000; Hardy and Vekemans, 2002; Rousset and Leblois, 2011). For diploid individuals in a twodimensional habitat, NS is equal to 1 divided by the slope of the regression between individual genetic distance $\left(a_{r}\right.$; Rousset, 2000) and the log of distance. With this estimate of NS and when the effective density of individuals $(D)$ is known, then

$$
\sigma=\sqrt{N S /(4 \pi D)}
$$

This approach provides a single estimate of NS across the entire population, rather than spatially explicit estimates of NS that we sought to produce. Moreover, inferring $\sigma$ from the equation above requires knowledge of $D$, which is readily quantified in simulations, but difficult to estimate in wild populations. The methods to infer $\sigma$ explored in this analysis do not require a priori knowledge of $D$.

In this analysis, we used a global population estimate of $\sigma$ (calculated from the mean squared dispersal distances in the simulations or estimated from $F_{I S}$ or HWE tests) to define analysis extents around sampled individuals. However, local variation in $\sigma$ could produce local neighborhoods that are not adequately defined by the global mean estimate. For example, dispersal distances could be influenced by local population density (Herzig, 1995). In such instances, estimating $\sigma$ locally for each neighborhood surrounding a sampled individual may be necessary.

Another important limitation of this approach applies only to populations isolated by resistance. In such cases, a resistance model is required to measure effective distances that define the extent of neighborhoods, yet these are available for relatively few species. Moreover, error in a resistance model would be manifest in inter-individual effective distances that define analysis neighborhood boundaries, potentially adding error or bias to local estimates of NS. Accurately quantifying landscape effects on genetic isolation is a primary focus in the field of landscape genetics. Methods have now been developed to parameterize resistance models based on maximizing the relationship between genetic relatedness and various hypotheses of how the landscape resists gene flow (Cushman et al., 2006, 2013; Shirk et al., 2010). As advances are made in this field and as empirical resistance models become increasing available, the potential for spatial analysis of $N S$ in IBR populations will grow.

Importantly, the implications of violating Wright's genetic neighborhood assumptions have not been explored as they relate to the spatially explicit method we describe. Wright's neighborhood concept assumes a uniform population density and Gaussian dispersal. However, in wild populations, dispersal has often been observed to be leptokurtic (Kot et al., 1996) and population density often varies over space and time. RobledoArnuncio and Rousset (2010) examined bias and error in the regression-based estimate of NS described above (Rousset, 2000) as a function of population aggregation and dispersal kurtosis. They found the method was robust to departures from Gaussian dispersal and population aggregation as long as NS was interpreted as the speed of genetic differentiation with distance (not the number of effective individuals within a radius of $2 \sigma$ ) and $\sigma^{2}$ was interpreted as the asymptotic mean rate of increase in meansquared dispersal distance of a gene lineage. Whether the spatially explicit estimates of NS provided by our approach is similarly robust needs to be evaluated in populations exhibiting spatial demographic variability and non-Gaussian dispersal.

\section{CONCLUSIONS}

Existing methods to quantify NS in continuous populations produce a single estimate reflecting the aggregate behavior of all neighborhoods in the population. We have shown the potential for profound spatial variation in NS that is masked by the population mean estimate and described a method to detect this variation (implemented in the R package sGD). The magnitude 
of spatial variation in NS may be sufficient to create conditions for inbreeding depression at the periphery or in areas isolated by strong patterns of resistance, even though the core remains viable. This raises the potential for a spatial component to extinction vortex dynamics in continuous populations. Spatially explicit estimates of NS may be used to asses genetic viability risk patterns across a landscape, thereby aiding conservation genetic analyses and management. However, the method we propose is predicated on sufficient sampling density, an accurate estimate of $\sigma$, an accurate landscape genetic model (IBD or IBR), and assumptions of Wright's neighborhood that may be violated in real populations (e.g., Gaussian dispersal). New studies that examine the accuracy of this method under variable sample size and population demographic qualities are needed.

\section{ACKNOWLEDGMENTS}

We thank R. Waples and three anonymous reviewers for helpful comments on the manuscript.

\section{DATA ARCHIVING}

This article does not report new empirical data. The $\mathrm{R}$ package sGD is freely available to download from http://cran. r-project.org.

\section{REFERENCES}

Amos, W., and Harwood, J. (1998). Factors affecting levels of genetic diversity in natural populations. Philos. Trans. R. Soc. Lond. B Biol. Sci. 353, 177-186.

Aspi, J., Roininen, E., Ruokonen, M., Kojola, I., and Vila, C. (2006). Genetic diversity, population structure, effective population size and demographic history of the Finnish wolf population. Mol. Ecol. 15, 1561-1576. doi: 10.1111/j.1365294X.2006.02877.x

Caballero, A. (1994). Developments in the prediction of effective population size. Heredity 73, 657-679.

Caughley, G. (1994). Directions in conservation biology. J. Anim. Ecol. 63, 215-244.

Cockerham, C. C., and Weir, B. S. (1977). Digenic descent measures for finite populations. Genet. Res. 30, 121-147.

Cushman, S. A., McKelvey, K. S., Hayden, J., and Schwartz, M. K. (2006). Gene flow in complex landscapes: testing multiple hypotheses with causal modeling. Am. Nat. 168, 486-499. doi: 10.1086/506976

Cushman, S. A., Wasserman, T. N., Landguth, E. L., and Shirk, A. J. (2013). Reevaluating causal modeling with mantel tests in landscape genetics. Diversity 5 , 51-72. doi: 10.3390/d5010051

Do, C., Waples, R. S., Peel, D., Macbeth, G. M., Tillett, B. J., and Ovenden, J. R. (2014). NeEstimator V2: re-implementation of software for the estimation of contemporary effective population size (Ne) from genetic data. Mol. Ecol. Resour. 14, 209-214. doi: 10.1111/1755-0998.12157

Eckert, C. G., Samis, K. E., and Lougheed, S. C. (2008). Genetic variation across species' geographical ranges: the central-marginal hypothesis and beyond. Mol. Ecol. 17, 1170-1188. doi: 10.1111/j.1365-294X.2007.03659.x

Frankham, R., Briscoe, D. A., and Ballou, J. D. (2002). Introduction to Conservation Genetics. Cambridge, UK: Cambridge University Press. doi: 10.1017/CBO9780 511808999

Franklin, I. R. (1980). "Evolutionary change in small populations," in Conservation Biology: An Evolutionary-Ecological Perspective, eds M. E. Soulé, and B. A. Wilcox (Sunderland, MA: Sinauer Associates), 135-149.

García-Ramos, G., and Kirkpatrick, M. (1997). Genetic models of adaptation and gene flow in peripheral populations. Evolution 51, 21-28.

Gardner, R. H. (1999). "RULE: map generation and spatial analysis program," in Landscape Ecological Analysis: Issues and Applications, eds J. M. Klopatek and R. H. Gardner (New York, NY: Springer-Verlag), 280-303. doi: 10.1007/978-14612-0529-6_13

Goslee, S. C., and Urban, D. L. (2007). The ecodist package for dissimilarity-based analysis of ecological data. J. Stat. Softw. 22, 1-19.
Hardy, O. J., and Vekemans, X. (1999). Isolation by distance in a continuous population: reconciliation between spatial autocorrelation analysis and population genetics models. Heredity 83, 145-154.

Hardy, O. J., and Vekemans, X. (2002). SPAGeDi: a versatile computer program to analyse spatial genetic structure at the individual or population levels. Mol. Ecol. Notes 2, 618-620. doi: 10.1046/j.1471-8286.2002.00305.x

Herzig, A. L. (1995). Effects of population density on long-distance dispersal in the goldenrod beetle Trirhabda virgata. Ecology 76, 2044-2054.

Hill, W. G. (1981). Estimation of effective population size from data on linkage disequilibrium. Genet. Res. 38, 209-216. doi: 10.1017/S0016672300020553

Ibrahim, K. M., Nichols, R. A., and Hewitt, G. M. (1996). Spatial patterns of genetic variation generated by different forms of dispersal. Heredity 77, 282-291.

Kot, M., Lewis, M. A., and van Den Driessche, P. (1996). Dispersal data and the spread of invading organisms. Ecology 77, 2027-2042.

Lande, R., and Barrowclough, G. F. (1987). "Effective population size, genetic variation, and their use in population management," in Viable Populations for Conservation, ed M. E. Soulé (Cambridge, UK: Cambridge University Press), 87-123.

Landguth, E. L., and Cushman, S. A. (2010). CDPOP: a spatially explicit cost distance population genetics program. Mol. Ecol. Resour. 10, 156-161. doi: 10.1111/j.1755-0998.2009.02719.x

Maruyama, T. (1972). Rate of decrease of genetic variability in a two-dimensional continuous population of finite size. Genetics 70, 639-651.

McRae, B. H. (2006). Isolation by resistance. Evolution 60, 1551-1561. doi: 10.1554/05-321.1

Miller, M. P. (2005). Alleles in space (AIS): computer software for the joint analysis of interindividual spatial and genetic information. J. Heredity 96, 722-724. doi: 10.1093/jhered/esi119

Neel, M. C., McKelvey, K. S., Ryman, N., Lloyd, M. W., Short Bull, R., Allendorf, F. W., et al. (2013). Estimation of effective population size in continuously distributed populations: there goes the neighborhood. Heredity 111, 189-199. doi: 10.1038/hdy.2013.37

Pudovkin, A., Zaykin, D., and Hedgecock, D. (1996). On the potential for estimating the effective number of breeders from heterozygote-excess in progeny. Genetics 144, 383-387.

R Development Core Team. (2013). R: A Language and Environment for Statistical Computing. R Foundation for Statistical Computing. Vienna: R Development Core Team.

Reed, D. H., and Bryant, E. H. (2000). Experimental tests of minimum viable population size. Anim. Conserv. 3, 7-14. doi: 10.1017/S136794300000069X

Reed, D. H., and Frankham, R. (2001). How closely correlated are molecular and quantitative measures of genetic variation? a meta-analysis. Evolution 55, 1095-1103. doi: 10.1554/0014-3820(2001)055[1095:HCCAMA]2.0.CO;2

Robledo-Arnuncio, J. J., and Rousset, F. (2010). Isolation by distance in a continuous population under stochastic demographic fluctuations. J. Evol. Biol. 23 , 53-71. doi: 10.1111/j.1420-9101.2009.01860.x

Rohlf, F. J., and Schnell, G. D. (1971). An investigation of the isolation-by-distance model. Am. Nat. 105, 295-324.

Rousset, F. (2000). Genetic differentiation between individuals. J. Evol. Biol. 13, 58-62. doi: 10.1046/j.1420-9101.2000.00137.x

Rousset, F., and Leblois, R. (2011). Likelihood-based inferences under isolation by distance: two-dimensional habitats and confidence intervals. Mol. Biol. Evol. 29, 957-973. doi: 10.1093/molbev/msr262

Shirk, A. J., and Cushman, S. A. (2011). sGD: software for estimating spatially explicit indices of genetic diversity. Mol. Ecol. Resour. 11, 922-934. doi: 10.1111/j.1755-0998.2011.03035.x

Shirk, A. J., Wallin, D. O., Cushman, S. A., Rice, C. G., and Warheit, K. I. (2010) Inferring landscape effects on gene flow: a new model selection framework. Mol. Ecol. 19, 3603-3619. doi: 10.1111/j.1365-294X.2010.04745.x

Smouse, P. E., and Peakall, R. (1999). Spatial autocorrelation analysis of individual multiallele and multilocus genetic structure. Heredity 82, 561-573.

Sokal, R. R., and Wartenberg, D. E. (1983). A test of spatial autocorrelation analysis using an isolation-by-distance model. Genetics 105, 219-237.

van Etten, J. (2011). Gdistance: Distances and Routes on Geographical Grids. R package version 1.1-2.

Wahlund, S. (1928). The combination of populations and the appearance of correlation examined from the standpoint of the study of heredity. Hereditas 11, 65-106. 
Wang, J. (2009). A new method for estimating effective population sizes from a single sample of multilocus genotypes. Mol. Ecol. 18, 2148-2164. doi: 10.1111/j.1365-294X.2009.04175.x

Wang, J. L., and Caballero, A. (1999). Developments in predicting the effective size of subdivided populations. Heredity $82,212-226$.

Waples, R. S. (1989). A generalized-approach for estimating effective populationsize from temporal changes in allele frequency. Genetics 121, 379-391.

Waples, R. S. (2006). A bias correction for estimates of effective population size based on linkage disequilibrium at unlinked gene loci. Conserv. Genet. 7, 167-184. doi: 10.1007/s10592-005-9100-y

Waples, R. S., and Do, C. (2008). LDNE: a program for estimating effective population size from data on linkage disequilibrium. Mol. Ecol. Resour. 8, 753-756. doi: 10.1111/j.1755-0998.2007.02061.x

Waples, R. S., and Do, C. (2010). Linkage disequilibrium estimates of contemporary $\mathrm{N}(\mathrm{e})$ using highly variable genetic markers: a largely untapped resource for applied conservation and evolution. Evol. Appl. 3, 244-262. doi: 10.1111/j.17524571.2009.00104.x

Wasserman, T. N., Cushman, S. A., Littell, J. S., Shirk, A. J., and Landguth, E. L. (2013). Population connectivity and genetic diversity of American marten (Martes americana) in the United States northern Rocky Mountains in a climate change context. Conserv. Genet. 14, 529-541. doi: 10.1007/s10592-012-0336-z

Weir, B. S. (1996). Genetic Data Analysis II: Methods for Discrete Population Genetic Data. Sunderland, MA: Sinauer Associates.
Wright, S. (1931). Evolution in mendelian populations. Genetics 16:97.

Wright, S. (1943). Isolation by distance. Genetics 28, 114-138.

Wright, S. (1946). Isolation by distance under diverse systems of mating. Genetics $31: 39$.

Conflict of Interest Statement: The authors declare that the research was conducted in the absence of any commercial or financial relationships that could be construed as a potential conflict of interest.

Received: 21 May 2014; accepted: 18 September 2014; published online: 08 October 2014.

Citation: Shirk AJ and Cushman SA (2014) Spatially-explicit estimation of Wright's neighborhood size in continuous populations. Front. Ecol. Evol. 2:62. doi: 10.3389/ fevo.2014.00062

This article was submitted to Evolutionary and Population Genetics, a section of the journal Frontiers in Ecology and Evolution.

Copyright (C) 2014 Shirk and Cushman. This is an open-access article distributed under the terms of the Creative Commons Attribution License (CC BY). The use, distribution or reproduction in other forums is permitted, provided the original author(s) or licensor are credited and that the original publication in this journal is cited, in accordance with accepted academic practice. No use, distribution or reproduction is permitted which does not comply with these terms. 